\title{
Direct patterning of probe proteins on an antifouling PLL-g-dextran coating for reducing the background signal of fluorescent immunoassays
}

\author{
Amandine MC Egea ${ }^{1,2,3}$, Emmanuelle Trévisiol ${ }^{1,4,5,6}$ and Christophe Vieu ${ }^{1,3,7^{*}}$
}

\begin{abstract}
The limit of detection of advanced immunoassays, biochips and micro/nano biodetection devices is impacted by the non-specific adsorption of target molecules at the sample surface. In this paper, we present a simple and versatile low cost method for generating active surfaces composed of antibodies arrays surrounded by an efficient anti-fouling layer, capable to decrease drastically the fluorescence background signal obtained after interaction with a solution to be analyzed. The technological process involves the direct micro-contact printing of the antibodies probe molecules on a pre-coated PLL-g-dextran thin layer obtained by contact printing using a flat PDMS stamp. Compared to other blocking strategies (ethanolamine blocking treatment, PLL-g-PEG incubation, PLL-g-dextran incubation, printing on a plasma-deposited PEO layer), our surface chemistry method is more efficient for reducing non-specific interactions responsible for a degraded signal/noise ratio.
\end{abstract}

Keywords: Microcontact printing; Surface patterning; Antifouling coating; Immunoassays; PLL-g-dextran

\section{Background}

Protein microarrays are used to detect and semi-quantify proteins in solution and have been used for a variety of applications (Coleman et al. 2007; Gao et al. 2005; Popescu et al. 2007; Zhu et al. 2000). Antibody microarray, seen as a multiplexed immunoassay, is the most common type of protein chips, where antibodies are used as capture molecules to detect the concentrations of various antigens in solution (Kingsmore 2006). Antibody microarrays target essentially clinical diagnostics and proteomics fields, but their contribution is limited by: the availability of high-affinity and high-specificity antibodies for the capture of protein biomarkers, the possible protein denaturation and spatial configuration when grafted at the chip surface and finally, the non-specific adsorption of target proteins on the surface (Hucknall et al. 2009a). This last point directly impacts the biosensing performances, especially the limit of detection (LOD) (Hucknall et al. 2009b). Indeed, the minimal detected concentration of an immunoassay depends on the sensitivity of the signal

\footnotetext{
* Correspondence: cvieu@laas.fr

'CNRS, LAAS, 7 avenue du colonel Roche, F-31400 Toulouse, France

${ }^{3}$ CNRS, ITAV-UMS3039, F-31106 Toulouse, France

Full list of author information is available at the end of the article
}

used to transduce the antibody/antigen interaction but also to the signal/noise ratio, which strongly depends on the background signal coming from the area around the affinity spots. When fluorescence is used as an optical detection method, the practical limit of detection can thus be drastically improved by minimizing the background emission of the regions around the interaction spots. This background signal is caused by non-specifically adsorbed labelled antigens at the chip surface. Controlling protein adsorption is therefore essential to improve the sensitivity of immunoassays such as protein microarrays. With the development of biodetection based on nanosystems, the need for advanced surface chemistries capable to prevent non-specific adsorption events is becoming even more crucial, due to the extreme sensitivity of the transduction mechanism involved in these highly miniaturized devices (Krishnan et al. 2008). In a broader perspective, protein resistant or antifouling surfaces are of great interest for a variety of biomedical and biotechnological applications (Balamurugan et al. 2005; Chang et al. 2008; Chapman et al. 2001; Herrwerth et al. 2003; Nath et al. 2004; Ostuni et al. 2001; Schwendel et al. 2001). The strategies for reducing the background signal commonly involve a "blocking" step wherein the surface is exposed to a 
high-concentration of a blocking molecule that does not generate any detection signal. This passivating agent is intended to bind to any available surface interaction site, thereby restricting further nonspecific adsorption of the protein of interest. Among them, proteins such as Bovine Serum Albumin (BSA) or small molecules such as ethanolamine, are commonly used to reduce the background signal of biosensor based assays (Butler 2000). Polymer coatings are also widely used with a similar objective. Poly(L-Lysine)-graft-Poly(Ethylene Glycol) (PLL-g-PEG) (Kenausis et al. 2000; Morgenthaler et al. 2006; Pasche et al. 2003) and more recently, poly(L-lysine)-graft-dextran (PLL-g-dextran) (Perrino et al. 2008) have been shown to prevent the non-specific adsorption of proteins. PLL-g-PEG is a graft copolymer consisting of PEG chains grafted onto a PLL polycationic backbone. The positive charges present on the protonated primary amine groups of the PLL backbone in neutral aqueous environment provides immobilization on negatively charged surfaces, via electrostatic interactions. Under optimized conditions the PEG chains adopt a brush conformation, which induces a steric repulsion preventing any interaction between proteins and the surface. This expected effect depends on many parameters such as the length, flexibility, and density of the PEG chains (Halperin 1999; Jeon \& Andrade 1991; Jeon et al. 1991; Mcpherson et al. 1995; Szleifer 1997; Zhu et al. 2001). PLL-g-dextran is a quite similar graft copolymer with dextran side chains onto a poly-L-lysine (PLL) backbone. Dextran is a natural polysaccharide consisting of an $\alpha$ - $(1,6)$-linked glucan with side chains attached to the 3-positions of the backbone glucose units. The dextran groups provide the required antifouling properties (Holland et al. 1998; Martwiset et al. 2006), which depend on its weight (Martwiset et al. 2006), structure (Osterberg et al. 1995) and grafting density.

Finally, for any kind of selected surface chemistry, the common challenge for the design of proteins microarrays is the robust immobilization of probe proteins of high affinity with the target molecule on very localized areas, while preserving an antifouling property of the overall chip surface. This engineering of the adsorption properties of a chip is achieved by surface functionalization using molecular patterning techniques (Zhou et al. 2011). From the micro to the nanoscale, various techniques were used for biopatterning purposes (Jackman et al. 1995; Wilbur et al. 1994; Xia et al. 1995). Among them, microcontact printing $(\mu \mathrm{CP})$ (Osterberg et al. 1995; Kumar \& Whitesides 1993; Ruiz \& Chen 2007; Xia \& Whitesides 1998a) turned out to display many advantages because of its simplicity, low cost, pattern definition, and large area processing (Michel et al. 2001; Quist et al. 2005). Combining $\mu \mathrm{CP}$ of probe molecules with antifouling layers has thus been investigated as a functionalization process for biological applications. Ruiz et al.
(Ruiz \& Chen 2007; Ruiz et al. 2007) reported that the direct printing of fibronectin and PLL on an anti-adhesive Poly Ethylene Oxide (PEO) film was possible. Indeed, it appeared that the PECVD (Plasma-Enhanced Chemical Vapour Deposition) deposited PEO layer (Bretagnol et al. 2006a), had the property of being protein-resistant and cell repellent in solution but protein-adhesive under the dry conditions used during the microcontact printing procedure (Ruiz et al. 2007; Delamarche et al. 2003). The PLL patterns on the PEO coating in these conditions were stable for several weeks (Rossi \& Colpo 2010), as revealed by fluorescence imaging after immersion in water for one month. We estimate that this direct patterning method greatly simplifies the fabrication of advanced biodetection chips and lead to much more controlled surfaces at the molecular scale because many incubations and blocking steps are avoided. To our mind and our experience, this advantage becomes crucial for biodetection nanodevices that are intrinsically very sensitive to any unavoidable adsorption events taking place at the solid-liquid interface, during the various incubation and washing steps required in more conventional protocols.

In this perspective, we have investigated if direct printing of probe proteins by $\mu \mathrm{CP}$ on a PLL-g-dextran antifouling coating is possible. Hence, anti-GST antibodies $(25 \times 25$ spots) were dry-printed on a PLL-g-dextran pre-coated slide. Biodetection experiments with target molecules (fluorescent-tagged GST) were then conducted by fluorescence. To evaluate the antifouling properties of the PLL-g-dextran surface, a systematic comparison with others surface chemistries (ethanolamine blocking, PLL-gPEG, PEO), also known in the literature to reduce the non-specific adsorption, was performed.

\section{Methods}

a. Glass slides processing

Glass slides (Gold Seal) were cleaned in an isopropanol ultrasonic bath (35 kHz, $5 \mathrm{~min}$, room temperature) and a deionized water ultrasonic bath $(35 \mathrm{kHz}$, $5 \mathrm{~min}$, room temperature). They were then activated by a $\mathrm{O}_{2}$ plasma treatment (Teplas, $800 \mathrm{~W}, 5 \mathrm{~min}$, 1,5 mBar) to provide a better reactivity during the subsequent surface chemistry protocols (Rossi \& Colpo 2010).

b. Coating of the antifouling PLL-g-dextran layer For coating the glass slides with a high quality PLLg-dextran homogeneous layer we deposit this polymer by $\mu$ CP rather than through an incubation step. For this purpose, we employ a flat un-patterned stamp made of Poly-DiMethyl-Siloxane (PDMS, Sylgard 184, Dow Corning, USA), molded on a perfectly flat silicon wafer, inked with a PLL-g-dextran solution and we transfer the polymer layer from the stamp to the plasma activated glass slide by contact. The PDMS 
stamp was produced using a mixture of Sylgard 184 and a curing agent, (10:1 ratio), which was poured on a flat silicon wafer previously functionalized with octadecyltrichlorosilane (OTS) to facilitate the demolding of the PDMS stamp. Cross-linking of PDMS was performed at $60^{\circ} \mathrm{C}$ for 6 hours. The PDMS stamp molded on the silicon wafer does not exhibit any topographical patterns at its surface (Figure 1A). This PDMS stamp was then gently activated by a $\mathrm{O}_{2}$ plasma treatment (Teplas, $300 \mathrm{~W}, 30 \mathrm{sec}, 1,5 \mathrm{mBar}$ ) and immediately inked with the PLL-g-dextran solution. The PLL(20 kDa)- $g$ (Popescu et al. 2007; Zhu et al. 2000)-dextran (5 kDa) polymer used in the following experiments was purchased at Susos, Switzerland. Phosphate-Buffered Saline (PBS) (0.01M PBS; $\mathrm{NaCl}$ $0.138 \mathrm{M}$; KCl 0.0027M; pH 7.4) was used to prepare the solution of polymer $(100 \mu \mathrm{g} / \mathrm{mL})$ which was stored at $-20^{\circ} \mathrm{C}$ before experiments. During the inking step, the PLL-g-dextran solution $(100 \mu \mathrm{g} / \mathrm{mL}$ in PBS 10 $\mathrm{mM}, \mathrm{pH}$ 7.4) was pipetted and incubated on the top of the flat PDMS stamp for $2 \mathrm{~min}$. (Figure 1B). The stamp was dried under nitrogen stream and brought in contact for $2 \mathrm{~min}$ with the activated glass slide. After stamp removal, a rapid washing step in a PBS solution under agitation (2 min, $1 \mathrm{mM}, \mathrm{pH} 7,4$ ) removed the weakly bounded polymer molecules, leaving an homogeneous high quality antifouling PLLg-dextran coating at the surface of the glass slide (Figure 1C).

c. Direct micro-contact printing of anti-GST antibodies on the PLL-g-dextran coating

A silicon master was structured by UV-photolithography on a positive resist (AZ1529) layer followed by Reactive Ion Etching (RIE) (Kumar \& Whitesides 1993; Xia \& Whitesides 1998a; Bernard et al. 1998; Delamarche et al. 1997; Xia \& Whitesides 1998b; Zhao et al. 1997). After an anti-adhesive treatment using OTS, a PDMS mixture is deposited on the silicon master. The cross-linked PDMS stamps exhibited geometric features of $160 \mu \mathrm{m}$ wide spots at a $320 \mu \mathrm{m}$ pitch (Figure 1D).

An anti-GST antibodies solution $(25 \mu \mathrm{g} / \mathrm{mL}$ in PBS, $\mathrm{pH} 7.4,1 \mathrm{mM}$ ) was pipetted and incubated on the top of the patterned PDMS stamp for $2 \mathrm{~min} 30 \mathrm{sec}$. (Figure 1E). The inked stamp was then dried under a stream of nitrogen and brought in contact for $2 \mathrm{~min}$ with the PLL-g-dextran pre-coated slide. Anti-GST antibodies were transferred from the PDMS stamp to the antifouling layer following the patterns designed on the stamp (Figure 1F), leading to an array of anti-GST antibodies spots patterned onto an antifouling PLL-g-dextran coating.

d. Preparation of comparative chips based on ethanolamine blocking, PLL-g-PEG coating after printing, PLL-g-dextran coating after printing and PEO coating before printing

Ethanolamine blocking solution: Epoxide-coated slides (Corning) were used. An array of anti-GST antibodies was patterned at the slide surface through $\mu \mathrm{CP}$ using the same conditions as before (see experimental section, part c). The slide was then immersed in an ethanolamine solution (Tris- $\mathrm{HCl}, 200 \mathrm{mM}, \mathrm{pH} \mathrm{8,2)} \mathrm{for}$ 30 min under agitation at room temperature and finally washed in a PBS solution $(10 \mathrm{mM}, 3 \times 5 \mathrm{~min}$, $\mathrm{pH} \mathrm{7,4).}$

PLL-g-PEG antifouling coating after probe molecules printing: An unfunctionalized glass slide was cleaned and activated (experimental section part a). An array of anti-GST antibodies was then patterned at the slide surface through $\mu C P$ (experimental section, part c). A PLL-g-PEG solution $(100 \mu \mathrm{g} / \mathrm{mL}$ in PBS, $10 \mathrm{mM}, \mathrm{pH} 7,4)$ was then incubated on the slide for 4 min at room temperature and a washing step in a PBS solution (10 mM, $3 \mathrm{~min}, \mathrm{pH} \mathrm{7,4)}$ was finally performed. The PLL-g-PEG solution was prepared with PLL(20 kDa)-g(Popescu et al. 2007; Zhu et al. 2000)-PEG(5 kDa) molecules, purchased at Susos, Switzerland, in PBS.




PLL-g-dextran antifouling coating after probe molecules printing: An unfunctionalized glass slide was cleaned and activated (experimental section, part a). An array of anti-GST antibodies was patterned on the slide through $\mu C P$ (experimental section, part c). A PLL-g-dextran solution $(100 \mu \mathrm{g} / \mathrm{mL}$ in PBS, 10 $\mathrm{mM}, \mathrm{pH} \mathrm{7,4)}$ was incubated on the slide for $4 \mathrm{~min}$ at room temperature and a washing step in a PBS solution (3 min, $1 \mathrm{mM}, \mathrm{pH} 7,4$ ) was finally performed.

PEO coating before probe molecules printing: The PEO-like layer is produced by plasma polymerization (Bretagnol et al. 2006a; Bretagnol et al. 2006b; Bretagnol et al. 2007) on a glass slide and provided by the European Commission, Joint Research Centre, Institute for Health and Consumer Protection, TP 203. Via Fermi, 21027 Ispra (VA), Italy. The array of anti-GST antibodies is then directly patterned on the PEO pre-coated slide through $\mu \mathrm{CP}$ (see experimental section, part c).

e. Interaction with Cy3-labelled GST

Target molecules (Cy3-labelled Glutathione-STransferase) were purchased at Tebu-bio. A solution of Cy3-labelled GST ( $5 \mu \mathrm{g} / \mathrm{mL}$ in PBS, $10 \mathrm{mM}$, $\mathrm{pH}$ 7.4) was incubated on the various functionalized slides for 30 minutes at room temperature. A washing step was then achieved $(3 \times 5 \mathrm{~min}$ in PBS $10 \mathrm{mM}+$ $0,1 \%$ tween 20). Fluorescence analyses were performed to quantify the adsorption of the target molecules onto the chip surface and evaluate the efficiency of the surface chemistry to minimize the background signal.

f. Characterization technique

Fluorescence quantifications were performed using an InnoScan 900 scanner, dedicated to microarrays measurements (Innopsys, France). This equipment composed of two wavelengths $(\lambda=532 \mathrm{~nm}, \lambda=635$ $\mathrm{nm}$ ) exhibits a spatial resolution of $1 \mu \mathrm{m}$. We measured the surface fluorescence intensities, thanks to the image treatment software Mapix (Innopsys, France). The fluorescent signal between the antibodies spots (i.e. background) is measured and systematically compared after statistical analysis for the various surface chemistries tested in this work.

\section{Results and discussion}

Microcontact printing with a flat PDMS stamp was used to coat a protein-resistant layer composed of PLL-gdextran. This method of deposition was preferred to a direct incubation of PLL-g-dextran on the slide surface as it provides thickness homogeneity over large areas. Indeed, the printed PLL-g-dextran layer reaches $4 \mathrm{~nm}$ of thickness as attested by Atomic Force Microscoscopy (AFM) measurements in liquid environment, (see Additional file 1 for AFM measurements) which was performed at different sites of the glass slide. An array of anti-GST antibody (Ab) spots was then immobilized through $\mu \mathrm{CP}$ on the antifouling PLL-g-dextran coating. The surface is therefore composed of $160 \mu \mathrm{m}$ wide antibody printed spots spaced by $160 \mu \mathrm{m}$ areas of antifouling PLL-g-dextran. A solution of Cy3-labelled GST was then incubated for 30 minutes on these bi-functionalized surfaces alternating high affinity regions (printed $\mathrm{Ab}$ spots) with very low affinity spaces (PLL-g-dextran film). Figure 2 displays a fluorescence image after interaction showing a clear spatial localization of the GST labelled-molecules precisely on the antibody printed spots. The fluorescent areas accurately match the geometry of the probe patterns (spot of $160 \mu \mathrm{m}$ ). The image size shows excellent reproducibility of the patterns over a $\mathrm{cm}^{2}$ area, and pattern homogeneity was obtained over an entire glass slide. After a complete immunoassay protocol, the probe molecules patterned on the PLL-g-dextran layer attended to be stable after several washing steps. Furthermore, the fluorescence intensity of the background is close to zero, showing that the antifouling printed PLL-g-dextran layer significantly reduces the non-specific adsorption on the surface between the $\mathrm{Ab}$ printed spots. Such a micro-engineered substrate thus offers localized areas where proteins attachment is robust while non-specific adsorption is inhibited on the rest of the surface. This result shows that direct contact printing of Abs can be performed at the dry state by $\mu \mathrm{CP}$ on the PLL-g-dextran coating. During the contact printing, the thin polymer film is not in a brush configuration because the PEG chains are not sufficiently hydrated. The probe molecules are thus correctly transferred from the

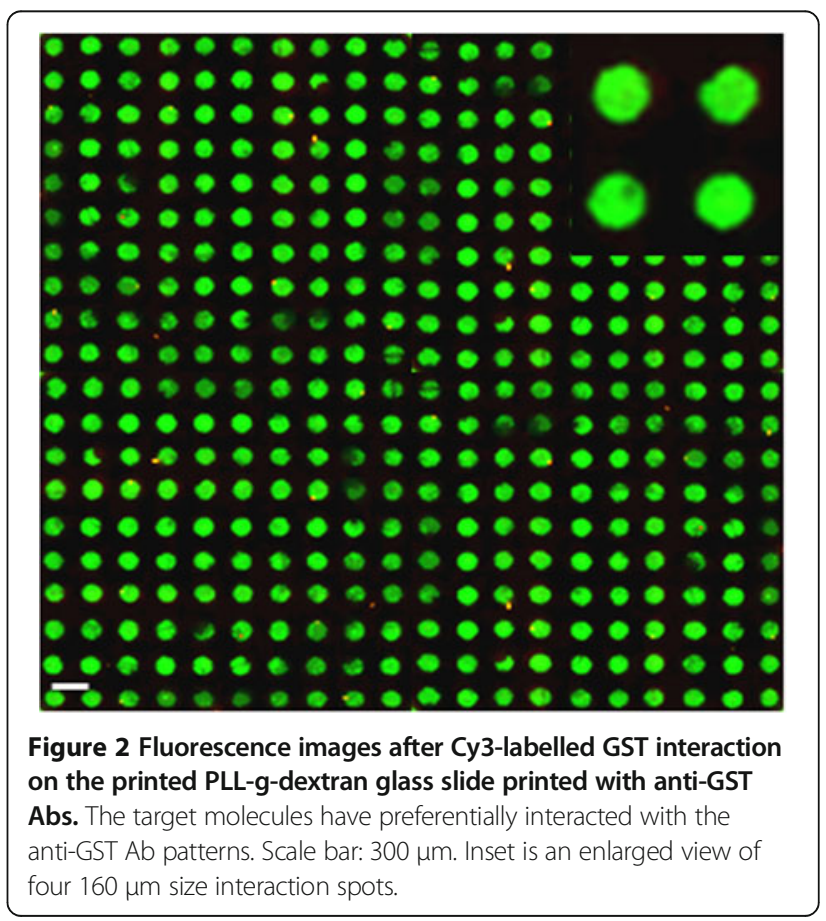


PDMS stamp to the surface and establish stable and robust interactions with the substrate. When the surface is later incubated with a solution of the target labelled-GST proteins, only the PLL-g-dextran molecules located in the regions in-between the printed spots, recover their brush configuration in solution, conferring an antifouling property to these zones. The PLL-g-dextran molecules just below the printed Abs cannot change their molecular configuration and the Ab spots remains robustly attached on the surface. This experiment reproduces quite well the results obtained on the PEO layers (Ruiz \& Chen 2007; Ruiz et al. 2007; Bretagnol et al. 2006a; Bretagnol et al. 2006b; Bretagnol et al. 2007; Bretagnol et al. 2006c).

To quantify the antifouling performance of this PLLg-dextran printed surface and confirm that our choice to print directly the probe molecules on a pre-coated PLL-g-dextran surface was optimal, we have compared the background levels obtained for alternative surface chemistries already proposed in the literature for reducing the background of fluorescent immunoassays. For all the investigated conditions, the fluorescent characterizations clearly showed a spatial localization of the GST labelled-molecules on the anti-GST antibodies spots, which exhibited a fluorescent signal significantly higher than the background signal. The results showing the background signal are displayed in Figure 3. The error bars display the background signal dispersion over the slide. As a control experiment, we have printed the Ab probe molecules on a plasma-activated glass slide and performed the interaction with the target solution. As expected in this case, we observe a significant background signal (1670 u.a.) (Figure 3), due to the non-specific adsorption of GST labelled-proteins at the glass surface.
Any other surface chemistry protocol of the glass slide needs to show lower levels of background fluorescence as compared to this rough reference. In the case of a conventional blocking protocol involving incubation in ethanolamine solution after probe molecules patterning, the background signal can be reduced to (740 u.a.) indicating that, as expected, this strategy decreases the non-specific adsorption of target labelled-proteins. Another strategy, very often employed for combining $\mu \mathrm{CP}$ and anti-fouling coating, relies on the incubation of an anti-fouling polymer solution after the print of the probe molecules. In that case, we can expect that the polymeric anti-fouling layer assembled only around the deposited Ab printed patterns. We have tested this solution for two candidate polymers: PLL-g-PEG and PLL-g-dextran. Both protocols exhibited greater performance for decreasing the background signal, as compared to a blocking protocol with ethanolamine. In the case of a post-printing incubation of PLL-g-PEG, the signal around the interaction spots was as low as 620 u.a. and for a post-printing incubation of PLL-g-dextran, the background signal is only 360 u.a. For PLL-g-PEG incubation, a quite large dispersion of the results is observed attesting that the anti-fouling coating obtained by incubation after the print of the probe molecules is not homogenous or that some Ab probe can be desorbed from the printed spots and randomly occupy on the surface. In the two last conditions, we wanted to compare two strategies involving the direct printing of the probe Abs at the surface of a pre-coated anti-fouling polymer layer. Using a PECVD deposited PEO polymer coating we were able to decrease the background signal down to 150 u.a. which is approximately ten times lower than the control glass slide. This result confirms that the direct print of probe molecules on



Figure 3 Background fluorescence intensity after Cy3-labelled GST interaction. Five typical surface chemistry protocols: an epoxide treated glass slide saturated with an ethanolamine solution after anti-GST printing (1), a glass slide treated after anti-GST printing with a PLL-g-PEG solution (2), a glass slide treated after anti-GST printing with a PLL-g-dextran solution (3), a glass slide with anti-GST Abs directly printed on a PECVD deposited PEO layer (4) and a glass slide with anti-GST Abs directly printed on a PLL-g-dextran layer obtained by contact printing using a flat stamp according to the protocol explained in Figure 1 (5). An $\mathrm{O}_{2}$ plasma activated glass slide is used as a reference. 
this plasma deposited layer is an efficient strategy, as already described by A. Ruiz et al. In our study, a background signal of 116 u.a. is recorded when the print of the $\mathrm{Ab}$ probe molecules is achieved directly on a PLLg-dextran thin coating layer, previously deposited by contact printing using a flat PDMS stamp. The dispersion of the background signal over an entire glass slide is around $10 \%$ of the mean average: 16 u.a., which shows that this method generates an efficient anti-fouling layer of substantial homogeneity. This deposition process is more straightforward as compared to a PECVD process and gives superior performances in terms of decrease of the background signal of protein chips.

\section{Conclusions}

An advanced surface chemistry was experimented to prevent non-specific adsorption events on a biochip surface and decrease substantially the background signal of fluorescent immunoassays. An array of anti-GST Abs spots was transferred thought direct Micro-Contact printing on an anti-adhesive coating composed of printed PLL-gdextran molecules. This original strategy was inspired from thin PEO films deposited by PECVD, which for the first time were shown to be protein adhesive under the dry conditions of $\mu \mathrm{CP}$ and protein resistant in solution. In our methodology, the first PLL-g-dextran coating is obtained by contact printing using a flat stamp, which is a very low-cost, reproducible and fast method for generating this efficient anti-fouling coating as compared to more advanced methods such as PECVD deposition. Our process involving two contact printing steps, offers localized areas where proteins attachment is robust while the non-specific adsorption is inhibited on the rest of the surface. More than $90 \%$ of the non-specific protein adsorption can be eliminated with this process in comparison with an untreated glass slide. Compared to other blocking strategies known in the literature these advanced biochips appear more effective for reducing unspecific interactions around the affinity spots. This easy-to-use functionalization technique opens an interesting surface chemistry for improving the limit of detection of immunoassays and the biosensing performances of micro and nano biodetection devices.

\section{Additional file}

Additional file 1: Liquid Atomic Force Microscopy (AFM) measurements of a PLL-g-dextran cross-meshed patterns to confirm the thickness and homogeneity of the antifouling polymer coating obtained by contact printing with a plasma activated PDMS stamp.

\section{Competing interests}

The authors declare that they have no competing interests.

\section{Author's contributions}

AE conceived the experiments, prepared the biomolecular coatings and patterns and monitored the experiments. AE, ET and CV wrote the manuscript. All authors read and approved the final manuscript.

\section{Acknowledgements}

We thank the clean room facility of LAAS-CNRS for the fabrication of silicon molds used for the printing process. We greatly thank P. Colpo from JRC, Ispra Italy for the fabrication of the plasma deposited PEO films used in this study. This work was financially supported by the ERANET project DETECTMUNE financed by the Midi-Pyrénées regional council.

\section{Author details}

${ }^{1}$ CNRS, LAAS, 7 avenue du colonel Roche, F-31400 Toulouse, France. ${ }^{2}$ Innopsys, Parc d'Activités Activestre, 31390 Carbonne, France. ${ }^{3}$ CNRS, ITAV-UMS3039, F-31106 Toulouse, France. “Université de Toulouse; INSA, UPS, INP; LISBP, 135 Av. de Rangueil, F-31077 Toulouse, France. ${ }^{5}$ INRA, UMR792 Ingénierie des Systèmes Biologiques et des Procédés, F-31400 Toulouse, France. ${ }^{6}$ CNRS, UMR5504, F-31400 Toulouse, France. ${ }^{7}$ Univ de Toulouse, INSA, LAAS, F-31400 Toulouse, France.

Received: 16 July 2013 Accepted: 23 September 2013

Published: 31 December 2013

\section{References}

Balamurugan S, Ista LK, Yan J, Lopez GP, Fick J, Himmelhaus M, Grunze M (2005) Reversible protein adsorption anf bioadhesion on monolayers terminated with mixtures of oligo(ethylene glycol) and methyl groups. J Am Chem Soc 127:14548-14549

Bernard A, Delamarche E, Schmid H, Michel B, Bosshard HR, Biebuyck H (1998) Printing patterns of proteins. Langmuir 14:2225-2229

Bretagnol F, Lejeune M, Papadopoulou-Bouraoui A, Hasiwa M, Rauscher H, Ceccone G, Colpo P, Rossi F (2006a) Fouling and non-fouling surfaces produced by plasma polymerization of ethylene oxide monomer. Acta Biomater 2:165-172

Bretagnol F, Ceriotti L, Lejeune M, Papadopoulou-Bouraoui A, Hasiwa M, Gilliland D, Ceccone G, Colpo P, Rossi F (2006b) Functional micropatterned surfaces by combination of plasma polymerization and lift-off processes. Plasma Process Polym 3:30-38

Bretagnol F, Valsesia A, Ceccone G, Colpo P, Gilliland D, Ceriotti L, Hasiwa M, Rossi F (2006c) Surface functionalization and pPatterning techniques to design interfaces for biomedical and biosensor applications. Plasma Process Polym 3:443-455

Bretagnol F, Kylian O, Hasiwa M, Ceriotti L, Rauscher H, Ceccone G, Gilliland D, Colpo P, Rossi F (2007) Micro-patterned surfaces based on plasma modification of PEO-like coating for biological applications. Sensor Actuat B-Chem 123:283-292

Butler JE (2000) Solid supports in enzyme-linked immunosorbent assay and other solid-phase immunoassays. Methods 22:4-23

Chang Y, Liao S-C, Higuchi A, Ruaan R-C, Chu C-W, Chen W-Y (2008) A highly stable nonbiofouling surface with well-packed grafted zwitterionic polysulfobetaine for plasma protein repulsion. Langmuir 24:5453-5458

Chapman RG, Ostuni E, Liang MN, Meluleni G, Kim E, Yan L, Pier G, Warren HS, Whitesides GM (2001) Polymeric thin films that resist the adsorption of proteins and the adhesion of bacteria. Langmuir 17:1225-1233

Coleman MA, Beernink PT, Camarero JA, Albala JS (2007) Applications of functional protein microarrays: identifying protein-protein interactions in an array format. Methods Mol Biol 385:121-130

Delamarche E, Bernard A, Schmid H, Michel B, Biebuyck H (1997) Patterned delivery of immunoglobulins to surfaces using microfluidic networks. Science 276:779-781

Delamarche E, Donzel C, Kamounah FS, Wolf H, Geissler M, Stutz R, Schmidt-Winkel P, Michel B, Mathieu HJ, Schaumburg K (2003) Microcontact printing using poly(dimethylsiloxane) stamps hydrophilized by poly(ethylene oxide) silanes. Langmuir 19:8749-8758

Gao WM, Kuick R, Orchekowski RP, Misek DE, Qiu J, Greenberg AK, Rom WN, Brenner DE, Omenn GS, Haab BB, Hanash SM (2005) Distinctive serum protein profiles involving abundant proteins in lung cancer patients based upon antibody microarray analysis. BMC Cancer 5:110

Halperin A (1999) Polymer brushes that resist adsorption of model proteins: design parameters. Langmuir 15:2525-2533

Herrwerth S, Eck W, Reinhardt S, Grunze M (2003) Factors that determine the protein resistance of oligoether self-assembled monolayers - internal hydrophilicity, 
terminal hydrophilicity, and lateral packing density. J Am chem soc 125:9359-9366

Holland NB, Qiu Y, Ruegsegger M, Marchant RE (1998) Biomimetic engineering of non-adhesive glycocalyx-like surfaces using oligosaccharide surfactant polymers. Nature 392:799-801

Hucknall A, Rangarajan S, Chilkoti A (2009a) In pursuit of zero: polymer brushes that resist the adsorption of proteins. Adv Mater 21:2441-2446

Hucknall A, Kim DH, Rangarajan S, Hill RT, Reichert WM, Chilkoti A (2009b) Simple fabrication of antibody microarrays on nonfouling polymer brushes with femtomolar sensitivity for protein analytes in serum and blood. Adv Mater 21:1968-1971

Jackman RJ, Wilbur JL, Whitesides GM (1995) Fabrication of submicrometer features on curved substrates by microcontact printing. Science 269:664-666 Jeon SI, Andrade JD (1991) Protein surface interactions in the presence of polyethylene oxide.2. Effect of protein size. J Colloid Interf Sci 142:159-166

Jeon SI, Lee JH, Andrade JD, Degennes PG (1991) Protein surface interactions in the presence of polyethylene oxide.1. Simplified theory. J Colloid Interf Sci 142:149-158

Kenausis GL, Vörös J, Elbert DL, Huang N, Hofer R, Ruiz-Taylor L, Textor M, Hubbell JA Spencer ND (2000) Poly(L-lysine)-g-poly(ethylene glycol) layers on metal oxide surfaces: attachment mechanism and effects of polymer architecture on resistance to protein adsorption. J Phys Chem B 104:3298-3309

Kingsmore SF (2006) Multiplexed protein measurement: technologies and applications of protein and antibody arrays. Nature reviews. Drug discov 5:310-320

Krishnan S, Weinman CJ, Ober CK (2008) Advances in polymers for anti-biofouling surfaces. J Mater Chem 18:3405-3413

Kumar A, Whitesides GM (1993) Features of gold having micrometer to centimeter dimensions Can Be formed through a combination of stamping with an elastomeric stamp and an alkanethiol Ink followed by chemical etching. Appl Phys Lett 63:2002-2004

Martwiset S, Koh AE, Chen W (2006) Nonfouling characteristics of dextrancontaining surfaces. angmuir 22:8192-8196

Mcpherson TB, Lee SJ, Park K (1995) Analysis of the prevention of protein adsorption by steric repulsion theory. Proteins Interfaces li 602:395-404

Michel B, Bernard A, Bietsch A, Delamarche E, Geissler M, Juncker D, Kind H, Renault JP, Rothuizen H, Schmid H, Schmidtwinkel P, Stutz R, Wolf H (2001) Printing meets lithography: soft approaches to high-resolution patterning. Ibm J Res Dev 45:870-870

Morgenthaler S, Zink C, Stadler B, Voros J, Lee S, Spencer ND, Tosatti SG (2006) Poly(L-lysine)-grafted-poly(ethylene glycol)-based surface-chemical gradients. Preparation, characterization, and first applications. Biointerphases 1:156-165

Nath N, Hyun J, Ma H, Chilkoti A (2004) Surface engineering strategies for control of protein and cell interactions. Surf Sci 570:98-110

Osterberg E, Bergstrom K, Holmberg K, Schuman TP, Riggs JA, Burns NL, Vanalstine JM, Harris JM (1995) Protein-rejecting ability of surface-bound dextran in End-on and side-on configurations - comparison to Peg. J Biomed Mater Res 29:741-747

Ostuni E, Chapman RG, Holmlin RE, Takayama S, Whitesides GM (2001) A survey of structure - property relationships of surfaces that resist the adsorption of protein. Langmuir 17:5605-5620

Pasche S, De Paul SM, Vörös J, Spencer ND, Textor M (2003) Poly (L-lysine)-graftpoly (ethylene glycol) assembled monolayers on niobium oxide surfaces: a quantitatitve study of the influence of polymer interfacial architecture on resistance to protein adsorption by ToF-SIMS ans in situ OWLS. Langmuir 19:9216-9225

Perrino C, Lee S, Choi SW, Maruyama A, Spencer ND (2008) A biomimetic alternative to poly(ethylene glycol) as an antifouling coating: resistance to nonspecific protein adsorption of poly(L-lysine)-graft-dextran. Langmuir 24:8850-8856

Popescu SC, Popescu GV, Bachan S, Zhang Z, Seay M, Gerstein M, Snyder M, Dinesh-Kumar SP (2007) Differential binding of calmodulin-related proteins to their targets revealed through high-density Arabidopsis protein microarrays. Proc Natl Acad Sci U S A 104:4730-4735

Quist AP, Pavlovic E, Oscarsson S (2005) Recent advances in microcontact printing. Anal Bioanal Chem 381:591-600

Rossi F, Colpo P (2010) Plasma processes and applications in NanoBiotechnology. J Phys Conf Ser 252:012001

Ruiz SA, Chen CS (2007) Microcontact printing: a tool to pattern. Soft Matter 3:168-177
Ruiz A, Ceriotti L, Buzanska L, Hasiwa M, Bretagnol F, Ceccone G, Gilliland D, Rauscher H, Coecke S, Colpo P, Rossi F (2007) Controlled micropatterning of biomolecules for cell culturing. Microelectron Eng 84:1733-1736

Schwendel D, Dahint R, Herrwerth S, Schloerholz M, Eck W, Grunze M (2001) Temperature dependence of the protein resistance of poly-and oligo(ethylene glycol)- terminated alkanethiolate monolayers. Langmuir 17:5717-5720

Szleifer I (1997) Protein adsorption on surfaces with grafted polymers: a theoretical approach. Biophys J 72:595-612

Wilbur JL, Kumar A, Kim E, Whitesides GM (1994) Microfabrication by microcontact printing of self-assembled monolayers. Adv Mater 6:600-604

Xia YN, Whitesides GM (1998a) Soft lithography. Angew Chem Int Edit 37:551-575

Xia YN, Whitesides GM (1998b) Soft lithography. Annu Rev Mater Sci 28:153-184

Xia Y, Mrksich M, Kim E, Whitesides GM (1995) Microcontact printing of octadecylsiloxane on the surface of silicon dioxide and its application in microfabrication. J Am Chem Soc 117:9576-9577

Zhao XM, Xia YN, Whitesides GM (1997) Soft lithographic methods for nano-fabrication. J Mater Chem 7:1069-1074

Zhou X, Boey F, Huo F, Huang L, Zhang H (2011) Chemically functionalized surface patterning. Small 16:2273-2289

Zhu H, Klemic JF, Chang S, Bertone P, Casamayor A, Klemic KG, Smith D, Gerstein M, Reed MA, Snyder M (2000) Analysis of yeast protein kinases using protein chips. Nat Genet 26:283-289

Zhu B, Eurell T, Gunawan R, Leckband D (2001) Chain-length dependence of the protein and cell resistance of oligo(ethylene glycol)-terminated self-assembled monolayers on gold. J Biomed Mater Res 56:406-416

doi:10.1186/1559-4106-8-37

Cite this article as: Egea et al:: Direct patterning of probe proteins on an antifouling PLL-g-dextran coating for reducing the background signal of fluorescent immunoassays. Biointerphases 2013 8:37.

\section{Submit your manuscript to a SpringerOpen ${ }^{\circ}$ journal and benefit from:}

- Convenient online submission

- Rigorous peer review

- Immediate publication on acceptance

- Open access: articles freely available online

- High visibility within the field

- Retaining the copyright to your article

Submit your next manuscript at $\gg$ springeropen.com 